\title{
Erratum to: Population and Computational Analysis of the MGEA6 P521A Variation as a Risk Factor for Familial Idiopathic Basal Ganglia Calcification (Fahr's Disease)
}

Roberta R. Lemos • Danyllo F. Oliveira • Mayana Zatz • João R. M. Oliveira

Published online: 29 January 2011

(C) Springer Science+Business Media, LLC 2011

Erratum to: J Mol Neurosci

DOI 10.1007/s12031-010-9445-7

The original version of this article unfortunately contained a mistake. First, the correct primer pair listed in the Methods and Sample Collection section is: (5'caaaatcaaaggatacattcagga 3 'forward) and (5' agctccttttccaaataaaagttatc 3'reverse). Secondly, Figure 1, the image associated with subject I-1 from family a, is an MRI image and not a CT image.

The online version of the original article can be found at http://dx.doi. org/10.1007/s12031-010-9445-7.

R. R. Lemos · D. F. Oliveira · J. R. M. Oliveira

Keizo Asami Laboratory (LIKA),

Federal University of Pernambuco,

Recife, Pernambuco, Brazil

M. Zatz

Human Genome Study Center, University of São Paulo,

São Paulo, São Paulo, Brazil

J. R. M. Oliveira $(\square)$

Neuropsychiatry Department,

Federal University of Pernambuco (UFPE),

50670901 Recife, Pernambuco, Brazil

e-mail: joao.ricardo@ufpe.br 